\title{
Modelo Estatístico do Algoritmo LMS de Erro Filtrado Modificado
}

\author{
Juan R. V. López, Orlando J. Tobias e Rui Seara
}

\begin{abstract}
Resumo-Este artigo propõe um modelo do algoritmo de erro filtrado modificado (MFeLMS). O modelo proposto é obtido sem invocar a teoria da independência, considerando adaptação lenta e sinais de entrada Gaussianos. Dado que o modelo em questão faz uso de uma recursividade de ordem elevada na descrição de seu comportamento, uma aproximação de terceira ordem através de truncamento é aqui considerada. Tal característica representa uma das principais contribuições deste trabalho. Simulações numéricas ratificam o bom casamento entre os resultados obtidos através do método de Monte Carlo e o modelo proposto, tanto para sinais de entrada brancos quanto coloridos.
\end{abstract}

Palavras-chave-Algoritmo LMS, algoritmo LMS de erro filtrado modificado, modelagem de algoritmos adaptativos.

Abstract-This paper proposes an improved stochastic model for the modified filtered-error least-mean-square (MFeLMS) algorithm. The proposed model is obtained without invoking the classic independence theory, and considering both a slow adaptation condition and Gaussian input signals. Since the model in question makes use of a high order recursive formulation to describe its behavior, a third-order approximation by using a truncation approach is here considered. Such a characteristic represents one of the main contributions of this work. Numerical simulations ratify the good agreement between the results obtained with the Monte Carlo method and the proposed model for both white and colored inputs.

Keywords-LMS algorithm, modified filtered-error LMS algorithm, adaptive algorithm modeling.

\section{INTRODUÇÃO}

Filtros adaptativos LMS vêm sendo utilizados satisfatoriamente em diferentes aplicações, tais como em equipamentos biomédicos, comunicações, controle ativo de ruído acústico e vibrações, dentre outras. Em algumas dessas aplicações, a implementação direta da estrutura adaptativa não é viável. Tal obstáculo é devido ao sinal de erro, utilizado para a atualização dos coeficientes, nem sempre estar disponível (acessível) para ser usado diretamente pelo algoritmo. Nesses casos, tem-se apenas uma versão filtrada ou atrasada do sinal de erro. Essa situação é comum em aplicações de controle ativo de ruído acústico e vibrações

Juan R. V. López e Rui Seara, LINSE-Laboratório de Circuitos e Processamento de Sinais, Depto. de Eng. Elétrica, Universidade Federal de Santa Catarina, Florianópolis, SC, E-mails: \{juan, seara\}@linse.ufsc.br.

Orlando J. Tobias, LINSE/UFSC e Departamento de Engenharia Elétrica e Telecomunicações, Universidade Regional de Blumenau, SC, E-mail: tobias.oj@ieee.org.

Este trabalho foi parcialmente financiado pela Coordenação de Aperfeiçoamento de Pessoal de Nível Superior (CAPES) e pelo Conselho Nacional de Desenvolvimento Científico e Tecnológico (CNPq). como também em cancelamento de eco acústico. Em tais situações, deve ser utilizado o algoritmo adaptativo de erro filtrado. No entanto, o fato de se ter uma versão filtrada ou atrasada do erro faz com que esse algoritmo apresente deficiências em relação à velocidade de convergência e estabilidade. Para contornar tais problemas, em [1], foi proposta uma modificação do algoritmo em questão, resultando em um melhor desempenho de convergência e estabilidade. A alteração é tal que o algoritmo modificado passa a ter um comportamento similar ao do algoritmo LMS convencional em termos de estabilidade e velocidade de convergência. Tal algoritmo é denominado algoritmo LMS de erro filtrado modificado (MFeLMS) [1], [2].

$\mathrm{Na}$ literatura, podemos encontrar três tipos de algoritmos pertencentes à família dos algoritmos adaptativos de erro filtrado. O LMS filtrado (FxLMS) [3]-[4], que é amplamente utilizado em aplicações de controle ativo de ruído acústico; o LMS filtrado modificado [2], que é uma versão modificada do FxLMS convencional; e o LMS de erro filtrado (FeLMS) [5]-[6], utilizado como uma implementação alternativa ao algoritmo FxLMS em cancelamento de eco [5] e equalização adaptativa [6], dentre outras aplicações.

Em geral, o algoritmo LMS e suas diferentes versões são modelados à luz da teoria da independência (TI) [7]-[8]. Entretanto, tal teoria não pode mais ser considerada em algumas situações, uma delas é o caso do algoritmo FxLMS. Um modelo para este último algoritmo é derivado em [9] e [10], resultando em um modelo muito mais preciso do que aquele obtido com a suposição de independência.

Neste trabalho, é proposto um modelo para o algoritmo MFeLMS, representando uma versão aprimorada daquele proposto em [11], que era baseado em uma aproximação de segunda ordem. Devido às características do algoritmo sob análise, a TI não é considerada aqui para derivar o modelo requerido. Os momentos de primeira e segunda ordens do vetor de coeficientes do algoritmo MFeLMS são determinados considerando uma condição de adaptação lenta e sinais de entrada Gaussianos. A comparação entre os resultados de simulação Monte Carlo mostram uma boa concordância com as predições obtidas a partir do modelo proposto, tanto para sinais de entrada brancos quanto coloridos.

\section{Algoritmo FeLMS CONVENCIONAL}

\section{A. Descrição do Algoritmo}

A Fig. 1 ilustra o diagrama de blocos do algoritmo FeLMS. A notação utilizada é: $\mathbf{w}_{\mathrm{o}}=\left[\begin{array}{llll}w_{\mathrm{o}, 0} & w_{\mathrm{o}, 1} & \cdots & w_{\mathrm{o}, N-1}\end{array}\right]^{\mathrm{T}}$ representa 
a resposta ao impulso da planta; $\mathbf{w}(n)=\left[w_{0}(n) w_{1}(n) \cdots\right.$ $\left.w_{N-1}(n)\right]^{\mathrm{T}}$ é o vetor de coeficientes adaptativos; $\mathbf{s}=\left[s_{0} s_{1}\right.$ $\left.\cdots s_{M-1}\right]^{\mathrm{T}}$ e $\hat{\mathbf{s}}=\left[\hat{s}_{0} \hat{s}_{1} \cdots \hat{s}_{\hat{M}-1}\right]^{\mathrm{T}}$ são, respectivamente, os coeficientes dos filtros representando o caminho de erro e sua estimativa; $d(n)$ e $z(n)$ denotam o sinal desejado e o ruído de medição, respectivamente. A variável $z(n)$ é um ruído de medição i.i.d, de média zero e descorrelacionado de qualquer outro de sinal no sistema. Nesta análise, o vetor de entrada é $\mathbf{x}(n)=[x(n) x(n-1) \cdots x(n-N+1)]^{\mathrm{T}}$, com $\{x(n)\}$ denotando um processo Gaussiano de média zero com variância $\sigma_{x}^{2}$. O vetor de entrada filtrado é dado por $\mathbf{x}_{\mathrm{f}}(n)=\left[x_{\mathrm{f}}(n)\right.$ $\left.x_{\mathrm{f}}(n-1) \cdots x_{\mathrm{f}}(n-N+1)\right]^{\mathrm{T}}$, onde

$$
\mathbf{x}_{\mathrm{f}}(n)=\sum_{i=0}^{\hat{M}-1} \hat{s}_{i} \mathbf{x}(n-i)
$$

Considera-se aqui que os vetores $\mathbf{w}_{\mathrm{o}}$ e $\quad \mathbf{w}(n)$ são de dimensões iguais; contudo, as dimensões dos vetores $\mathbf{s}$ e $\hat{\mathbf{s}}$ podem ser diferentes. Em geral, em uma aplicação prática, tem-se $M>\hat{M}$.

A partir da Fig. 1, o sinal de erro é dado por

$$
e(n)=d(n)-y(n)+z(n)
$$

onde $d(n)$ e $y(n)$ são obtidos como segue:

$$
d(n)=\mathbf{w}_{\mathrm{o}}^{\mathrm{T}} \mathbf{x}(n)=\mathbf{x}^{\mathrm{T}}(n) \mathbf{w}_{\mathrm{o}}
$$

e

$$
y(n)=\mathbf{w}^{\mathrm{T}}(n) \mathbf{x}(n)=\mathbf{x}^{\mathrm{T}}(n) \mathbf{w}(n) .
$$

Então, substituindo (4) em (2), obtém-se

$$
e(n)=d(n)-\mathbf{w}^{\mathrm{T}}(n) \mathbf{x}(n)+z(n) .
$$

O sinal de erro filtrado, usado para a atualização do vetor de coeficientes do algoritmo adaptativo, é dado por

$$
e_{\mathrm{f}}(n)=\sum_{i=0}^{M-1} s_{i} e(n-i) \text {. }
$$

Agora, substituindo (5) em (6), tem-se

$e_{\mathrm{f}}(n)=\sum_{i=0}^{M-1} s_{i} d(n-i)-\sum_{i=0}^{M-1} s_{i} \mathbf{w}^{\mathrm{T}}(n-i) \mathbf{x}(n-i)+\sum_{i=0}^{M-1} s_{i} z(n-i)$.

Assim, a expressão da atualização do vetor de coeficientes para o algoritmo FeLMS é dada por [12]

$$
\mathbf{w}(n+1)=\mathbf{w}(n)+\mu e_{\mathrm{f}}(n) \mathbf{x}_{\mathrm{f}}(n) .
$$

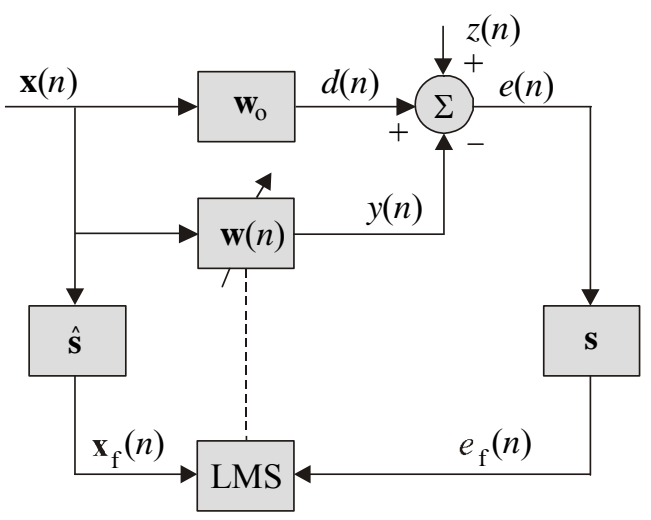

Fig. 1. Diagrama de blocos do algoritmo FeLMS.

\section{B. Algoritmo FeLMS Modificado}

O algoritmo MFeLMS é obtido pela compensação da operação de filtragem ocorrida no caminho do erro, representada pela expressão (6). Tal compensação é realizada adicionando o termo $-\Lambda_{\mathrm{f}}(n)$ em (7). Assim, o sinal de erro compensado é dado por [1]

$$
\begin{aligned}
\hat{e}_{\mathrm{f}}(n)= & \sum_{i=0}^{M-1} s_{i} d(n-i)-\sum_{i=0}^{M-1} s_{i} \mathbf{w}^{\mathrm{T}}(n-i) \mathbf{x}(n-i) \\
& +\sum_{i=0}^{M-1} s_{i} z(n-i)-\Lambda_{\mathrm{f}}(n) .
\end{aligned}
$$

Agora, a expressão da atualização do vetor de coeficientes é dada por

$$
\mathbf{w}(n+1)=\mathbf{w}(n)+\mu \hat{e}_{\mathrm{f}}(n) \mathbf{x}_{\mathrm{f}}(n) .
$$

O termo de compensação $\Lambda_{\mathrm{f}}(n)$ é obtido forçando que a expressão (9) seja igual ao sinal de erro do algoritmo LMS convencional [1]. Assim,

$$
\begin{aligned}
\left.e(n)\right|_{\mathrm{LMS}}= & d_{\mathrm{f}}(n)-\mathbf{w}^{\mathrm{T}}(n) \mathbf{x}_{\mathrm{f}}(n)+z_{\mathrm{f}}(n) \\
= & \sum_{i=0}^{M-1} s_{i} d(n-i)-\sum_{i=0}^{M-1} s_{i} \mathbf{w}^{\mathrm{T}}(n) \mathbf{x}(n-i) \\
& +\sum_{i=0}^{M-1} s_{i} z(n-i)
\end{aligned}
$$

Note que em (11) o sinal de erro agora depende do valor atual do vetor de coeficientes adaptativos. Igualando (9) e (11), o termo de compensação é obtido por

$$
\Lambda_{\mathrm{f}}(n)=\sum_{i=0}^{M-1} s_{i}\left[\mathbf{w}^{\mathrm{T}}(n)-\mathbf{w}^{\mathrm{T}}(n-i)\right] \mathbf{x}(n-i)
$$

A diferença $\left[\mathbf{w}^{\mathrm{T}}(n)-\mathbf{w}^{\mathrm{T}}(n-i)\right]$, em $(12)$, é obtida de (8), resultando em 


$$
\Lambda_{\mathrm{f}}(n)=\mu \sum_{i=0}^{M-1} \sum_{j=1}^{i} s_{i} \hat{e}_{\mathrm{f}}(n-j) \mathbf{x}_{\mathrm{f}}^{\mathrm{T}}(n-j) \mathbf{x}(n-i)
$$

Finalmente, substituindo (13) em (9), tem-se

$$
\begin{aligned}
\hat{e}_{\mathrm{f}}(n) & =\sum_{i=0}^{M-1} s_{i} d(n-i)-\sum_{i=0}^{M-1} s_{i} \mathbf{w}^{\mathrm{T}}(n-i) \mathbf{x}(n-i) \\
& -\mu \sum_{i=0}^{M-1} \sum_{j=1}^{i} s_{i} \hat{e}_{\mathrm{f}}(n-j) \mathbf{x}_{\mathrm{f}}^{\mathrm{T}}(n-j) \mathbf{x}(n-i)+\sum_{i=0}^{M-1} s_{i} z(n-i) .
\end{aligned}
$$

Note que em (14) o erro $\hat{e}_{\mathrm{f}}(n)$ depende do valor atual e valores passados; tal característica não se encontra presente no algoritmo FeLMS convencional. Entretanto, essa característica é a responsável em fazer com que o algoritmo modificado tenha um comportamento de convergência similar ao do algoritmo LMS convencional.

\section{MODELO}

\section{A. Considerações para Modelagem}

Para se obter uma expressão para o modelo proposto, as seguintes considerações são estabelecidas:

i) As correlações entre os diferentes vetores de entrada são muito mais importantes do que as correlações entre os vetores de entrada e os vetores de coeficientes.

ii) $\mathrm{O}$ modelo proposto é derivado considerando adaptação lenta; assim, os termos afetados por $\mu^{\beta} \operatorname{com} \beta \geq 4$ podem ser desconsiderados.

iii) Dado que o sinal de entrada é Gaussiano, os momentos de quarta e sexta ordens envolvendo o sinal de entrada são determinados através do teorema da fatoração de momentos [13].

\section{B. Comportamento Médio do Vetor de Coeficientes}

Nesta seção, é derivada uma expressão para o momento de primeira ordem dos coeficientes do filtro adaptativo. Assim, substituindo (1) e (14) em (10) e tomando o valor esperado em ambos os lados da expressão resultante, obtém-se

$$
\begin{aligned}
& E[\mathbf{w}(n+1)]=E[\mathbf{w}(n)]+\mu \sum_{i=0}^{M-1} \sum_{k=0}^{\hat{M}-1} s_{i} \hat{s}_{k} E[\mathbf{x}(n-k) d(n-i)] \\
& -\mu \sum_{i=0}^{M-1} \sum_{k=0}^{\hat{M}-1} s_{i} \hat{s}_{k} E\left[\mathbf{x}(n-k) \mathbf{w}^{\mathrm{T}}(n-i) \mathbf{x}(n-i)\right] \\
& -\mu^{2} \sum_{i=0}^{M-1} \sum_{j=1}^{i} \sum_{k=0}^{\hat{M}-1} s_{i} \hat{s}_{k} E\left[\mathbf{x}(n-k) \hat{e}_{\mathrm{f}}(n-j) \mathbf{x}_{\mathrm{f}}^{\mathrm{T}}(n-j) \mathbf{x}(n-i)\right] \\
& +\mu \sum_{i=0}^{M-1} \sum_{k=0}^{\hat{M}-1} s_{i} \hat{s}_{k} E[\mathbf{x}(n-k) z(n-i)] .
\end{aligned}
$$

As variáveis $d(n-i), \quad \mathbf{x}_{\mathrm{f}}^{\mathrm{T}}(n-j) \quad$ e $\quad \hat{e}_{\mathrm{f}}(n-j) \quad$ são determinadas de (3), (1) e (9), respectivamente; substituindo esses resultados em (15), usando as suposições (i)-(iii) e após uma manipulação algébrica, obtém-se

$$
\begin{aligned}
& E[\mathbf{w}(n+1)]=E[\mathbf{w}(n)]+\mu \sum_{i=0}^{M-1} \sum_{k=0}^{M} s_{i} \hat{s}_{k} \mathbf{R}_{i-k}\left\{\mathbf{w}_{\mathrm{o}}-E[\mathbf{w}(n-i)]\right\} \\
& -\mu^{2} \sum_{i=0}^{M-1} \sum_{j=1}^{i} \sum_{k=0}^{M} \sum_{l=0}^{M-1} \sum_{p=0}^{M-1} s_{i} \hat{s}_{k} s_{l} \hat{s}_{p}\left\{\mathbf{R}_{j+p-k} \mathbf{R}_{j+l-i}+\mathbf{R}_{i-k}\right. \\
& \left.\times \mathbf{R}_{l-p}+\mathbf{R}_{j+l-k} \operatorname{tr}\left[\mathbf{R}_{j+p-i}^{\mathrm{T}}\right]\right\}\left\{\mathbf{w}_{\mathrm{o}}-E[\mathbf{w}(n-j-l)]\right\} \\
& +\mu^{3} \sum_{i=0}^{M-1} \sum_{j=1}^{i} \sum_{k=0}^{\hat{M}-1} \sum_{l=0}^{M-1} \sum_{m=1}^{l} \sum_{p=0}^{\hat{M}-1} \sum_{q=0}^{M-1} \sum_{t=0}^{\hat{M}-1} s_{i} \hat{s}_{k} s_{l} \hat{s}_{p} s_{q} \hat{s}_{t} \\
& \times\left\{\mathbf{R}_{j+m+t-k} \mathbf{R}_{p-l} \mathbf{R}_{j+m+q-i}+\mathbf{R}_{j+m+t-k} \mathbf{R}_{i-j-l} \mathbf{R}_{m+q-p}\right. \\
& +\mathbf{R}_{j+m+t-k} \mathbf{R}_{m+q-l} \operatorname{tr}\left[\mathbf{R}_{i-j-p}\right]+\mathbf{R}_{j+l-k} \mathbf{R}_{p-m-t} \mathbf{R}_{j+m+q-i} \\
& +\mathbf{R}_{j+l-k} \mathbf{R}_{i-j-m-t} \mathbf{R}_{m+q-p}+\mathbf{R}_{j+l-k} \mathbf{R}_{q-t} \operatorname{tr}\left[\mathbf{R}_{i-j-p}\right] \\
& +\mathbf{R}_{j+p-k} \operatorname{tr}\left[\mathbf{R}_{l-m-t}\right] \mathbf{R}_{j+m+q-i}+\mathbf{R}_{j+p-k} \mathbf{R}_{i-j-m-t}^{\mathrm{T}} \mathbf{R}_{m+q-l} \\
& +\mathbf{R}_{j+p-k} \mathbf{R}_{i-j-l}^{\mathrm{T}} \mathbf{R}_{q-t}+\mathbf{R}_{i-k} \mathbf{R}_{p-l}^{\mathrm{T}} \mathbf{R}_{q-t} \\
& +\mathbf{R}_{i-k} \operatorname{tr}\left[\mathbf{R}_{m+t-l}\right] \mathbf{R}_{m+q-p}+\mathbf{R}_{i-k} \mathbf{R}_{m+t-p} \mathbf{R}_{m+q-l} \\
& +\mathbf{R}_{j+m+q-k} \operatorname{tr}\left[\mathbf{R}_{p-l} \mathbf{R}_{j+m+t-i}\right]+\mathbf{R}_{j+m+q-k} \operatorname{tr}\left[\mathbf{R}_{i-j-l} \mathbf{R}_{m+t-p}^{\mathrm{T}}\right] \\
& \left.+\mathbf{R}_{j+m+q-k} \operatorname{tr}\left[\mathbf{R}_{m+t-l}\right] \operatorname{tr}\left[\mathbf{R}_{i-j-p}^{\mathrm{T}}\right]\right\}\left\{\mathbf{w}_{o}-E[\mathbf{w}(n-j-m-q)]\right\} .
\end{aligned}
$$

As matrizes de autocorrelação em (16) são obtidas a partir da forma geral $\mathbf{R}_{\beta-\alpha}=E\left[\mathbf{x}(n-\alpha) \mathbf{x}^{\mathrm{T}}(n-\beta)\right]$.

\section{Valor de Regime Permanente de $\mathbf{w}(n)$}

Assumindo que o algoritmo converge, o valor em regime permanente do vetor de coeficientes é obtido a partir da condição

$$
\begin{aligned}
\lim _{n \rightarrow \infty} E[\mathbf{w}(n+1)] & =\lim _{n \rightarrow \infty} E[\mathbf{w}(n-i)]=\lim _{n \rightarrow \infty} E[\mathbf{w}(n-j-l)] \\
& =\lim _{n \rightarrow \infty} E[\mathbf{w}(n-j-m-q)]=\lim _{n \rightarrow \infty} E[\mathbf{w}(n)]=\mathbf{w}_{\infty} .
\end{aligned}
$$

Agora, aplicando (17) em (16) e considerando um problema de identificação de sistemas como ilustrado na Fig. 1, tem-se

$$
\mathbf{w}_{\infty}=\mathbf{w}_{\mathrm{o}} .
$$

A partir de (18), nota-se que o algoritmo MFeLMS tem o mesmo valor em regime permanente que o algoritmo FeLMS. É importante ressaltar que o termo de compensação apenas tem efeito durante a fase transiente do algoritmo. Tal fato pode ser melhor constatado através de (12), na qual se observa que na convergência a diferença $\left[\mathbf{w}^{\mathrm{T}}(n)-\mathbf{w}^{\mathrm{T}}(n-i)\right]$ é igual a zero. Portanto, é cancelado o efeito do termo de compensação $\Lambda_{\mathrm{f}}(n)$. 


\section{Curva de Aprendizagem}

Para determinar a expressão do modelo para a curva de aprendizagem do algoritmo MFeLMS, substitui-se (12) em (9) e, após algumas simples manipulações matemáticas, obtém-se

$$
\hat{e}_{\mathrm{f}}(n)=\sum_{i=0}^{M-1} s_{i} d(n-i)-\sum_{i=0}^{M-1} s_{i} \mathbf{w}^{\mathrm{T}}(n) \mathbf{x}(n-i)+\sum_{i=0}^{M-1} s_{i} z(n-i)
$$

Definindo então o vetor de erro nos coeficientes como sendo $\mathbf{v}(n)=\mathbf{w}(n)-\mathbf{w}_{\infty}$, onde $\mathbf{w}_{\infty}$ é o valor do vetor em regime permanente, dado por (18), e expressando (19) como uma função de $\mathbf{v}(n)$, elevando ao quadrado e tomando o valor esperado em ambos os lados da expressão resultante e aplicando as considerações de análise, tem-se

$$
\begin{aligned}
E\left[\hat{e}_{f}^{2}(n)\right]= & E\left[e_{\infty}^{2}(n)\right]-2 \sum_{i=0}^{M-1} s_{i} E\left[\hat{e}_{\infty}(n) \mathbf{v}^{\mathrm{T}}(n) \mathbf{x}(n-i)\right] \\
& +\sum_{i=0}^{M-1} \sum_{j=0}^{M-1} s_{i} s_{j} E\left\{\left[\mathbf{v}^{\mathrm{T}}(n) \mathbf{x}(n-i)\right]^{2}\right\}
\end{aligned}
$$

para

$$
\hat{e}_{\infty}(n)=\sum_{i=0}^{M-1} s_{i} d(n-i)-\sum_{i=0}^{M-1} s_{i} \mathbf{w}_{\infty}^{\mathrm{T}} \mathbf{x}(n-i)+\sum_{i=0}^{M-1} s_{i} z(n-i)
$$

onde $\hat{e}_{\infty}(n)$ representa o sinal de erro quando os coeficientes atingem o regime permanente.

Considerando que $E\left[\hat{e}_{\infty}(n) \mathbf{x}^{\mathrm{T}}(n-i)\right]=0$ (Princípio da Ortogonalidade [7]) e definindo $\xi_{\mathrm{f}}(n)=E\left[\hat{e}_{\mathrm{f}}^{2}(n)\right] \quad$ e $\xi_{\min }=E\left[\hat{e}_{\infty}^{2}(n)\right],(20)$ pode ser reescrita como segue:

$$
\xi_{\mathrm{f}}(n)=\xi_{\min }+\sum_{i=0}^{M-1} \sum_{j=0}^{M-1} s_{i} s_{j} \operatorname{tr}\left\{E\left[\mathbf{v}(n) \mathbf{v}^{\mathrm{T}}(n)\right] \mathbf{R}_{j-i}\right\}
$$

Para finalizar a derivação de (22), deve-se determinar o momento de segunda ordem de $\mathbf{v}(n)$. Para tal, já que o modelo é derivado considerando adaptação lenta, podemos utilizar a seguinte aproximação $E\left[\mathbf{v}(n) \mathbf{v}^{\mathrm{T}}(n)\right] \cong E[\mathbf{v}(n)]$ $E\left[\mathbf{v}^{\mathrm{T}}(n)\right][14]$.

\section{Resultados de Simulação}

Nesta seção, são apresentados dois exemplos para verificar o desempenho do modelo proposto em um problema de identificação de sistemas, considerando sinais de entrada Gaussianos. Para fins de comparação, são também mostrados alguns resultados obtidos a partir do modelo derivado usando a teoria clássica da independência [11].

\section{A. Exemplo 1}

Para este exemplo, a planta $\mathbf{w}_{\mathrm{o}}=[1,000,840,450,67$ $-0,19-0,470,390,120,30-0,54]^{\mathrm{T}}$ é considerada. $\mathrm{O}$ filtro no caminho do erro é dado por $\mathbf{s}=[0,0230,0710,194$ $0,3270,384]^{\mathrm{T}}$. O sinal de entrada é branco com variância $\sigma_{x}^{2}=1$. O máximo valor do passo de adaptação, determinado experimentalmente, para o qual o algoritmo converge é $\mu_{\max }=0,16$. O valor do passo de adaptação utilizado é $\mu=0,5 \mu_{\max }$. Os resultados de simulação são obtidos através do método de Monte Carlo (MC), considerando uma média de 100 realizações independentes. As Figs. 2 e 3 mostram, respectivamente, o comportamento médio dos coeficientes do filtro adaptativo (momento de primeira ordem) e a evolução do erro quadrático médio (EQM) (curva de aprendizagem) obtidos por simulação MC, modelo apresentado em [11], modelo obtido considerando a TI [11] (usado para fins de comparação) e modelo proposto [(16) e (22)]. Dessas figuras, observa-se que ambos os modelos (apresentado em [11] e proposto aqui) fornecem uma predição muito boa. Por outro lado, o modelo obtido a partir da TI apresenta um importante descasamento, principalmente na fase transiente. Nas Figs. 4 e 5, são considerados os mesmos parâmetros de simulação utilizados nas Figs. 2 e 3, porém agora considerando um valor de passo de adaptação $\mu=0,8 \mu_{\max }$. Nesse caso, observa-se que o modelo do algoritmo MFeLMS aqui proposto apresenta uma muito boa predição quando comparada com àquela do modelo sugerido em [11], tanto para o momento de primeira ordem (Fig. 4) quanto para a curva de aprendizagem (Fig. 5). Para uma melhor visualização, a Fig. 4 ilustra apenas dois coeficientes do filtro.

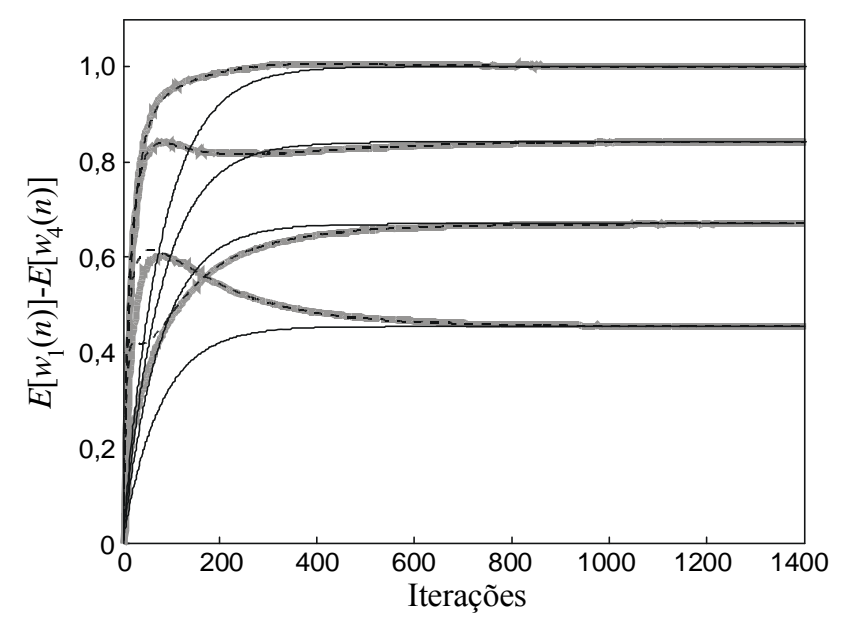

Fig. 2. Exemplo 1. Curvas do comportamento médio dos coeficientes $E\left[w_{1}(n)\right]$ até $E\left[w_{4}(n)\right]$ usando $\mu=0,5 \mu_{\max }$ para um sinal de entrada branco: simulação MC (linha cinza); modelo considerando a TI (linha contínua preta); para o modelo dado em [11] e o proposto em (16) (linha tracejada preta). 


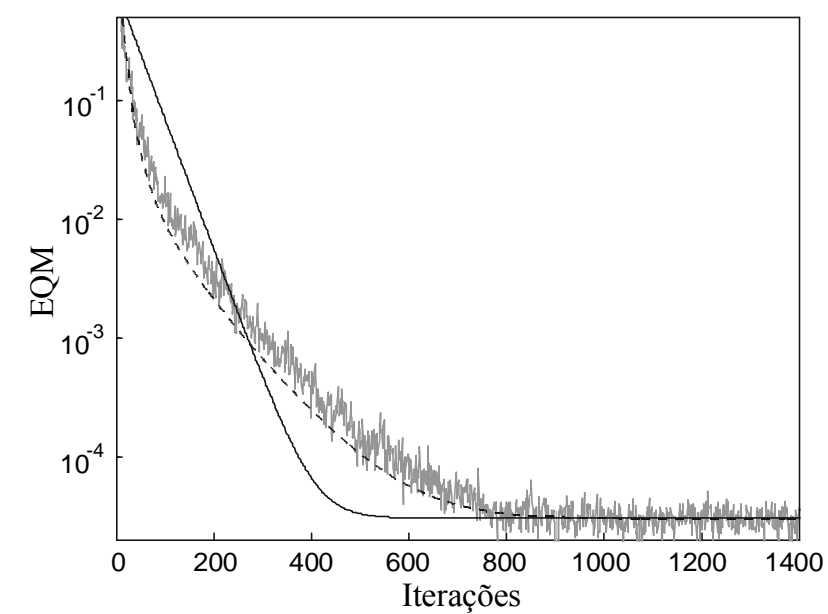

Fig. 3. Exemplo 1. Curvas do EQM usando $\mu=0,5 \mu_{\max }$ para um sinal de entrada branco: simulação MC (linha irregular cinza); modelo considerando a TI (linha contínua preta); para o modelo dado em [11] e o proposto em (22) (linha tracejada preta).

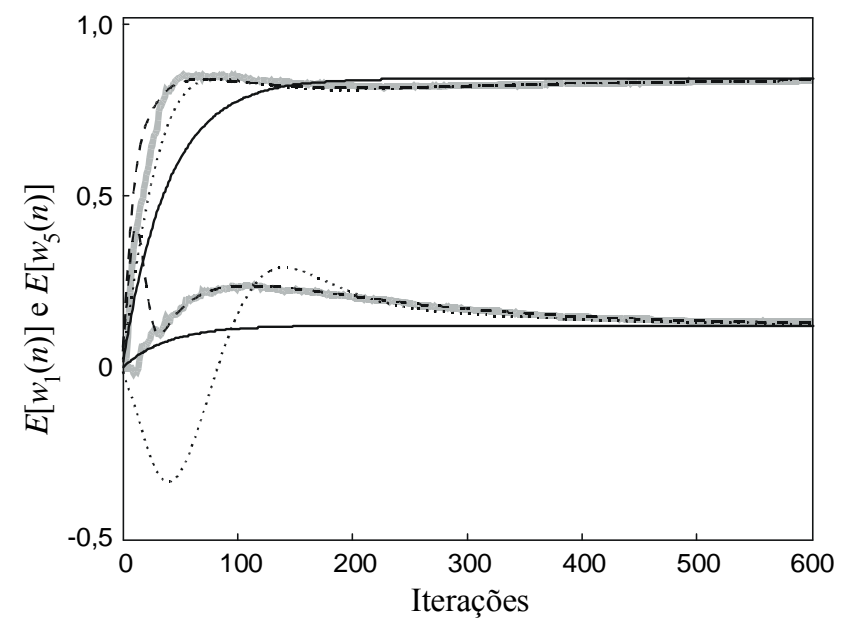

Fig. 4. Exemplo 1, agora considerando $\mu=0,8 \mu_{\max }$. Curvas do comportamento médio dos coeficientes $E\left[w_{1}(n)\right]$ e $E\left[w_{5}(n)\right]$ para um sinal de entrada branco: simulações MC (linha cinza); modelo usando a TI (linha contínua preta); modelo dado em [11] (linha pontilhada preta); modelo proposto em (16) (linha tracejada preta).

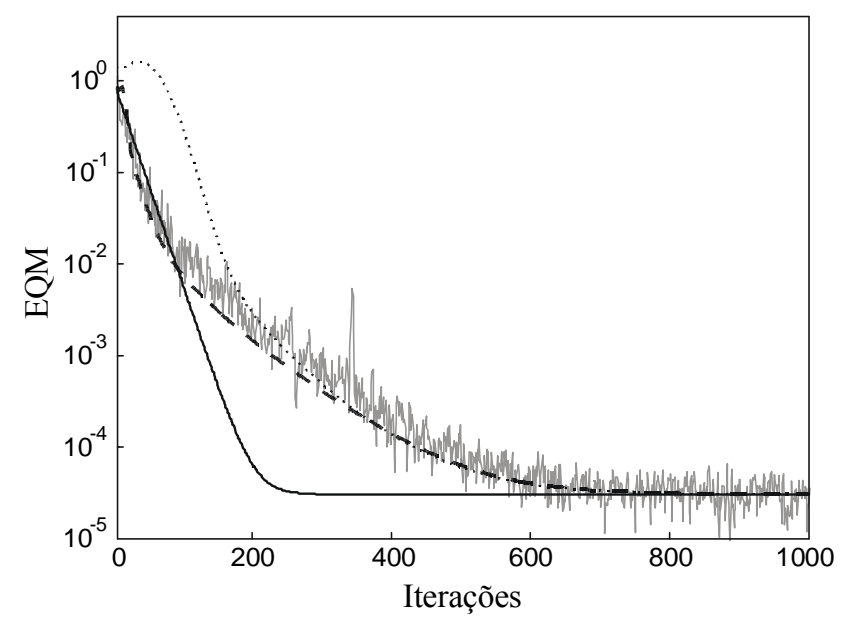

Fig. 5. Exemplo 1, agora para $\mu=0,8 \mu_{\max }$. Curvas do EQM para um sinal de entrada branco: simulação MC (linha irregular cinza); modelo usando a TI (linha contínua preta); modelo dado em [11] (linha pontilhada preta; modelo proposto em (22) (linha tracejada preta).

\section{B. Exemplo 2}

Neste caso, é usado sinal de entrada colorido, o qual é obtido de um processo AR(2) dado por

$$
x(n)=a_{1} x(n-1)+a_{2} x(n-2)+u(n)
$$

onde $u(n)$ é um ruído branco com variância unitária. Os coeficientes do processo $\operatorname{AR}(2)$ são $a_{1}=0,1833$ e $a_{2}=-0,75$, resultando em uma dispersão dos autovalores da matriz de autocorrelação do vetor de entrada igual a 26,87. A planta e os coeficientes do filtro no caminho do erro são os mesmos utilizados no exemplo anterior. O máximo passo de adaptação para este caso é $\mu_{\max }=0,2$ (determinado experimentalmente). $O$ passo de adaptação usado é $\mu=0,1 \mu_{\max }$. As Figs. 6 e 7 mostram, respectivamente, o comportamento do momento de primeira ordem e curva de aprendizagem obtidas por simulação $\mathrm{MC}$ e a partir dos modelos teóricos (usando a TI, apresentado em [11] e modelo proposto). Dessas figuras, é verificado um comportamento similar entre as aproximações de segunda ordem (modelo sugerido em [11]) e terceira ordem (modelo proposto) para sinais de entrada coloridos, independentemente do valor do passo de adaptação utilizado. Esse fato fica evidenciado nas Figs. 8 e 9; entretanto, o modelo usando a TI agora diverge.

Como comentário geral, observa-se que, para valores do passo de adaptação superior a $0,5 \mu_{\max }$ e sinais de entrada brancos, o modelo usando aproximação de terceira ordem fornece um melhor casamento com a simulação do que o modelo sugerido em [11]. Entretanto, quando utilizado um passo de adaptação inferior a $0,5 \mu_{\max }$, ambos os modelos (dado em [11] e proposto) apresentam um bom desempenho.

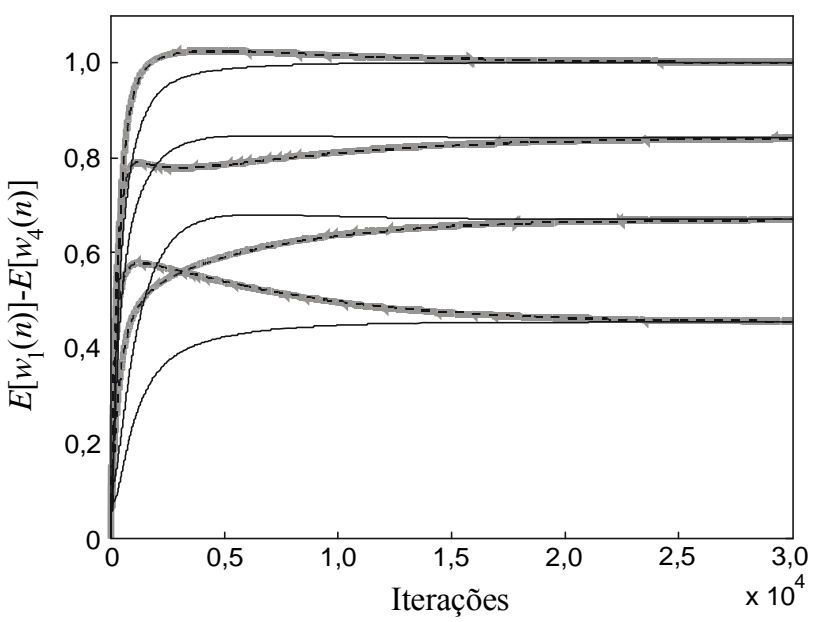

Fig. 6. Exemplo 2. Curvas do comportamento médio dos coeficientes $E\left[w_{1}(n)\right]$ até $E\left[w_{4}(n)\right]$ usando $\mu=0,1 \mu_{\max }$ para um sinal de entrada colorido: simulação MC (linha cinza); modelo considerando a TI (linha contínua preta); para o modelo dado em [11] e o proposto em (16) (linha tracejada preta). 


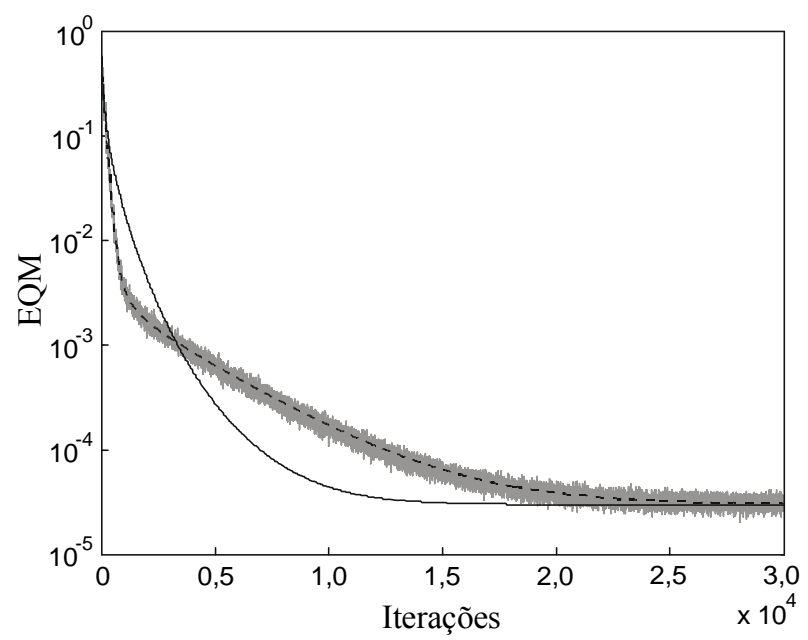

Fig. 7. Exemplo 2. Curvas do EQM usando $\mu=0,1 \mu_{\max }$ para um sinal de entrada colorido: simulação MC (linha irregular cinza); modelo considerando a TI (linha contínua preta); para o modelo dado em [11] e o proposto em (22) (linha tracejada preta).

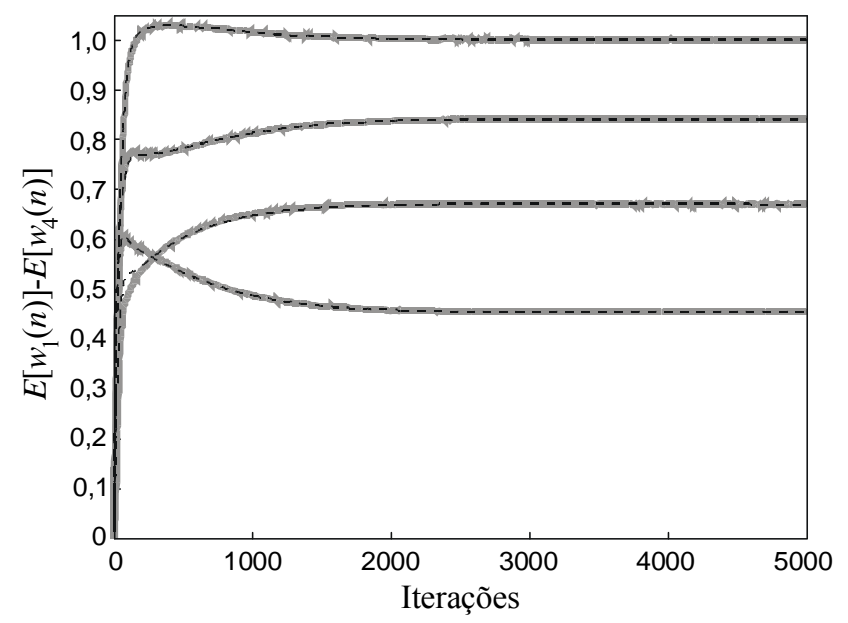

Fig. 8. Exemplo 2, agora considerando $\mu=\mu_{\max }$. Curvas do comportamento médio dos coeficientes $E\left[w_{1}(n)\right]$ até $E\left[w_{4}(n)\right]$ para um sinal de entrada colorido: simulação MC (linha cinza); para o modelo dado em [11] e o proposto em (16) (linha tracejada preta).

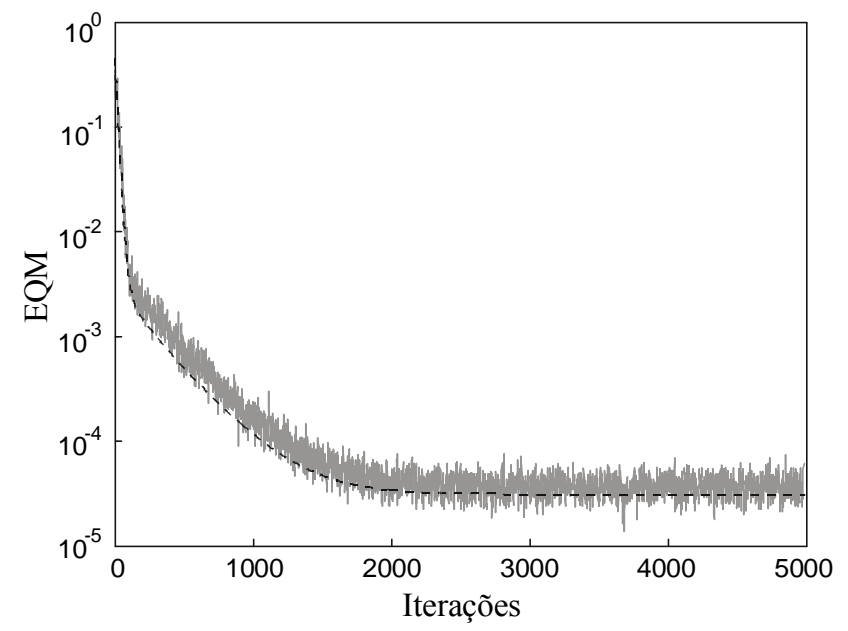

Fig. 9. Exemplo 2, considerando $\mu=\mu_{\max }$. Curvas do EQM para um sinal de entrada colorido: simulação MC (linha irregular cinza); para o modelo dado em [11] e o proposto em (22) (linha tracejada preta).

\section{CONCLUSÕES}

Neste trabalho, foi derivado um modelo para o algoritmo FeLMS modificado (MFeLMS) considerando adaptação lenta e não invocando a TI. Relativamente ao modelo proposto em [11], este trabalho considera uma aproximação de terceira ordem para obter a expressão (15), proporcionando uma maior precisão ao modelo proposto. Além do mais, foi também desenvolvido o equacionamento para momentos de sexta ordem de sinais Gaussianos, requeridos pela modelagem em questão. Simulações numéricas ratificaram o bom desempenho do modelo aqui apresentado tanto para sinais de entrada Gaussianos brancos quanto coloridos.

\section{REFERÊNCIAS}

[1] R. D. Poltmann, "Conversion of the delayed LMS algorithm into the LMS algorithm," IEEE Signal Process. Lett., vol. 2, no. 12, pp. 223, Dec. 1995.

[2] S. C. Douglas, "An efficient implementation of the modified filtered-x LMS algorithm," IEEE Signal Process. Lett., vol. 4, no. 10, pp. 286-288, Oct. 1997.

[3] S. J. Eliott and P. A. Nelson, "Active noise control," IEEE Signal Processing Mag., vol. 10, no. 4, pp. 12-35, Oct. 1993.

[4] J. R. Glover Jr., "Adaptive noise canceling applied to sinusoidal interferences," IEEE Trans. Acoust., Speech, Signal Process., vol. 25, no. 6, pp. 484-491, Dec. 1977.

[5] E. A. Wan, "Adjoint LMS: An efficient alternative to the filtered-x LMS and multiple error LMS algorithms," in Proc. IEEE Int. Conf. Acoust., Speech, Signal Process., Atlanta, USA, vol. 3, May 1996, pp. 1842-1845.

[6] S. Shaffer and C. S. Williams, "The filtered error LMS algorithm," in Proc. IEEE Int. Conf. Acoust., Speech, Signal Process., Boston, USA, Apr. 1983, pp. 4144.

[7] B. Widrow and S. D. Starns. Adaptive Signal Processing, Prentice Hall, 1998.

[8] E. Bjarnason, "Analysis of the filtered-X LMS algorithm," IEEE Trans. Speech and Audio Process., vol. 3, no. 6, pp. 504-514, Nov. 1995.

[9] O. J. Tobias, J. C. M. Bermudez, N. J. Bershad, and R. Seara, "Mean weight behavior of the filtered-X LMS algorithm," in Proc. IEEE Int. Conf. Acoust., Speech, Signal Process., Seattle, USA, May 1998, pp. 3545-3548.

[10] — "Second moment analysis of the filtered-X LMS algorithm," in Proc. IEEE Int. Conf. Acoust., Speech, Signal Process., Phoenix, USA, Mar. 1999, pp. 1873-1876.

[11] J. R. V. López, J. E. Kolodziej, O. J. Tobias, and R. Seara, "Statistical analysis of the first and second-order moments of the modified filtered error LMS algorithm," (in Portuguese) in Proc. XXII Brazilian Telecommunications Symposium, Campinas, SP, Brazil, Sep. 2005, pp. 593-598.

[12] E. Bjarnason, "Algorithms for active noise cancellation without exact knowledge of the error-path filter," in Proc. IEEE Int. Symp. Circ. Syst., London, UK, vol. 2, June 1994, pp. 573-576.

[13] N. Morita and T. Yajima, "Ultrahigh-time-resolution coherent transient spectroscopy with incoherent light," Phys. Rev. A, vol. 30, no. 5, pp. 2525-2536, Nov. 1984.

[14] N. J. Bershad, P. Celka, and J. M. Vesin, "Stochastic analysis of gradient adaptive identification of nonlinear systems with memory for Gaussian data and noisy input and output measurements," IEEE Trans. Signal Processing, vol. 47, no. 3, pp. 675-689, Mar. 1999. 